\title{
ON THE EXTENSOIN OF BERNOULLI, EULER AND EULERIAN POLYNOMIALS
}

\author{
S. N. SINGH AND S. S. MISHRA
}

\begin{abstract}
Here an attempt has been made to extend the Bernoulli, Euler and Eulerian polynomials in multiplication theorem and finite difference formula have been established.
\end{abstract}

\section{Introduction}

The study of Bernoulli, Euler and Eulerian polynomials has contributed much to our knowledge of the theory of numbers. These polynomials are of basic importance in several parts of Analysis and Calculus of Finite Differences and have application in various fields such as Statistics, Numerical Analysis etc. In recent years, the Eulerian numbers and Certain generalizations have been encountered in a number of Combinatorial problems (vide (1), (3), (4), (5), (6) for example). Of late, Singh and Rai (7) studied the extended polynomial set $B(n, h, a, k, x)$. This polynomial set was subjected to further investigation and Singh and Rai (8) succeeded in presenting novel two-variable extension of the same. A study of above polynomial set motivated us for consideration of following multi-variate extension (3.1) of the Bernoulli, Euler and Eulerian Polynomials and numbers as well as in the unified form from the point of view just described.

\section{Preliminary Results}

In 1964, Carlitz [2] extended the Bernoulli, Euler and Eulerian numbers and corresponding polynomials as

$$
\frac{\log \mathcal{G}(s)}{\mathcal{G}(S)-1}=\sum_{n=1}^{\infty} \frac{\beta(n)}{n^{s}}
$$

Received April 29, 1993; revised January 13, 1995.

1991 Mathematics Subject Classification. 11 B68.

Key words and phrases. Bernoulli, Euler, Eulerian polynomial, generating function, combinatories. 


$$
\begin{aligned}
\frac{(\mathcal{G}(s))^{z} \log \mathcal{G}(s)}{\mathcal{G}(s)-1} & =\sum_{n=1}^{\infty} \frac{\beta(n, z)}{n^{s}} \\
\frac{1-\lambda}{\mathcal{G}(s)-\lambda} & =\sum_{n=1}^{\infty} \frac{H(n, \lambda)}{n^{s}}, \quad \lambda \neq 1 \\
\frac{(1-\lambda) \mathcal{G}(s)^{z}}{\mathcal{G}(s)-\lambda} & =\sum_{n=1}^{\infty} \frac{H(n, \lambda, z)^{n}}{n^{s}}, \quad \lambda \neq 1
\end{aligned}
$$

When $\beta(n), \beta(n, z), H(n, \lambda)$ and $H(n, \lambda, z)$ are the extended Bernoulli and Eulerian numbers as well as corresponding polynomials. In the same paper, Carlitz considered a slightly more general situation, namely, the polynomials $\beta(n, h, z)$ and $H(n, h, \lambda, z)$ generated by

$$
\frac{h(\mathcal{G}(s))^{h z} \log \mathcal{G}(s)}{\left.(\mathcal{G}(S))^{h}-1\right)}=\sum_{n=1}^{\infty} \frac{\beta(n, h, z)}{n^{s}}
$$

and,

$$
\frac{(\lambda-1)(\mathcal{G}(s))^{h z}}{\left(\mathcal{G}(s)^{h}-\lambda\right)}=\sum_{n=1}^{\infty} \frac{H(n, h, \lambda, z)}{n^{s}}, \quad \lambda \neq 0, \lambda \neq 1
$$

It may be of interest to note that

$$
\epsilon(n, h, z)=H(n, h,-1, z)
$$

where $\epsilon(n, h, z)$ are extended Eulerian polynomials defined as

$$
\frac{2(\mathcal{G}(s))^{h z}}{\left((\mathcal{G}(s))^{h}+1\right)}=\sum_{n=1}^{\infty} \frac{\epsilon(n, h, z)}{n^{s}}
$$

It is familar that the formula

$$
\begin{gathered}
g(n)=\sum_{d / n} f(d), \quad(n=1,2,3, \ldots) \\
f(n)=\sum_{c d=n} \mu(c) g(d), \quad(n=1,2,3 \ldots)
\end{gathered}
$$

where $\mu(n)$ is Mobius function, are equivalent. If in (2.9) and (2.10), we take $n=$ $p_{1} p_{2} \ldots p_{r}$ where $p_{r}$ are distinct primes, it is easily verified that (2.9) and (2.10) reduce to

$$
\begin{gathered}
g_{r}=\sum_{j=0}^{r}\left(\begin{array}{l}
r \\
j
\end{array}\right) f_{j} ; \quad(r=0,1,2 \ldots) \\
f_{r}=\sum_{j=0}^{r}(-1)^{r-j}\left(\begin{array}{l}
r \\
j
\end{array}\right) g_{j} ; \quad(r=0,1,2, \ldots)
\end{gathered}
$$


respectively, where, for brevity, we put

$$
f_{r}=f\left(p_{1} p_{2} p_{3} \cdots p_{r}\right), \quad g_{r}=\left(p_{1} p_{2} p_{3} \cdots p_{r}\right)
$$

The equivalence of (2.11) and (2.12) is, of course, well known. The fact that the second equivalence is implied by first is perhaps not quite so familiar. It should be emphasized that $f(n)$ and $g(n)$ are arbitrary arithmetic functions subject only to $(2.9)$ or equivalently $(2.10)$, similar remark applies to $f_{r}$ and $g_{r}$.

Given a sequence

$$
f_{r} \quad(r=0,1,2, \ldots)
$$

we define an extended sequence

$$
f(n), \quad(n=0,1,2, \ldots)
$$

such that

$$
f\left(p_{1} p_{2} p_{3}, \cdots p_{r}\right)=f_{r}, \quad \text { where } p_{j} \text { are distinct prime; }
$$

Clearly, the extended sequence (2.14) is not uniquely determined by means of (2.15). If the sequence $g_{r}$ is related to $f_{r}$ by means of $(2.11)$, the sequence $g(n)$ defined by means of (2.9) furnishes an extension of the sequence $g_{r}$.

If we associate with the sequence $f_{r}$ the (formal) power series

$$
F_{t}=\sum_{r=0}^{\infty} f_{r} \frac{t^{r}}{r !}
$$

then (2.11) is equivalent to

$$
G_{t}=e^{t} F_{t}, \quad \text { where } \quad G(t)=\sum_{r=0}^{\infty} g_{r} \frac{t^{r}}{r !}
$$

We associate with the sequence $f(n)$ the (formal) Dirichlet series

$$
F(s)=\sum_{n=1}^{\infty} \frac{f_{n}}{n^{s}}
$$

Then (2.9) is equivalent to

$$
G(S)=\mathcal{G}(s) F(s)
$$

where

$$
G(s)=\sum_{n=1}^{\infty} \frac{g(n)}{n^{s}}, \quad \mathcal{G}(s)=\sum_{n=1}^{\infty} \frac{1}{n^{s}}
$$

It is well known that generating functions play an important role in the study of various useful properties of the polynomial sets which they generate. More systematic 
attacks have been made in this direction by Srivastava and Manocha [9]. The generating function concept led to the study of the following general class of polynomials.

\section{Extended Polynomial Set}

We define the polynomial set $P\left(n, h_{1}, h_{2}, \ldots, h_{k}, a_{1}, a_{2}, \ldots, a_{k}, m, i_{1}, i_{2}, \ldots, i_{k}, x_{1}\right.$, $\left.x_{2}, \ldots, x_{k}\right)$ by the following generating relation.

$$
\begin{aligned}
& \frac{m^{2}\left(\frac{h_{1}}{m} \log \mathcal{G}(s)\right)^{i_{1}}\left(\frac{h_{2}}{m} \log \mathcal{G}(s)\right)^{i_{2}} \cdots\left(\frac{h_{k}}{m} \log \mathcal{G}(s)\right)^{i_{k}}}{\left((\mathcal{G}(s))^{h_{1}}-a_{1}\right)\left((\mathcal{G}(s))^{h_{2}}-a_{2}\right) \cdots\left((\mathcal{G}(s))^{h_{k}}-a_{k}\right)} \times(\mathcal{G}(s))^{h_{1} x_{1}+h_{2} x_{2}+\cdots+h_{k} x_{k}} \\
= & \sum_{n=1}^{\infty} \frac{P\left(n, h_{1}, h_{2}, \ldots, h_{k}, a_{1}, a_{2}, \ldots a_{k}, m, i_{1}, i_{2}, \ldots, i_{k}, x_{1} x_{2}, \ldots x_{k}\right)}{n^{s}}
\end{aligned}
$$

Where $a_{1}, a_{2}, \ldots, a_{k}, m$ are non-zero real numbers, $i_{1}, i_{2}, \ldots, i_{k}$ are non-negative integers and $\left(h_{1}, h_{2}, \ldots, h_{k}\right) \neq 0$ and $\mathcal{G}(s)=\sum_{n=1}^{\infty} \frac{1}{n^{s}}$

We, now, notice following conditions

1. $i_{3}=i_{4}=\ldots i_{k}=0$

2. $h_{3}=h_{4}=\ldots h_{k}=1$ (Since, $\left.h_{1}, h_{2}, \ldots, h_{k} \neq 0\right)$

3. $a_{3}=a_{4}=\ldots a_{k}=0$ and

4. under the stated conditions, $\mathcal{G}(s)=1$, only when $\mathcal{G}(1)=1, n=1$.

If we apply above substitutions to the polynomial set $P\left(n, h_{1}, h_{2}, \ldots, h_{k}, a_{1}, a_{2}, \ldots a_{k}\right.$, $\left.m, i_{1}, i_{2}, \ldots, i_{k}, x_{1}, x_{2}, \ldots, x_{k}\right)$ this polynomial set reduces to polynomial set $P(n, h, k, a$, $b, m, i, j, x, y)$ defined by Singh and Rai [8]. Here, it is interesting to note that all the properties established for the polynomial set given by Singh and Rai [8] will spontaneously hold good for (3.1). In addition to these properties, some striking new properties will be set up. Categorically speaking, there will appear a plenty of properties in comparison to earlier.

The two-fold advantages of this may be explained: firstly, it is a generalized polynomial set in the sense that it unifies Bernoulli, Euler and Eulerian polynomials which, in turn, are obtainable from it on specializing various parameters involved therein; secondly, some striking new properties of these polynomials follow as direct consequences of itself.

We state the following relationships between our polynomial set and other polynomials

1. Extended Bernoulli Polynomials

Taking

$$
a_{1}=a_{2}=\cdots=a_{k-1}=a_{k}=i_{1}=i_{2}=\ldots=i_{k-1}=i_{k}=1
$$

we arrive at

$$
\begin{aligned}
& P\left(n, h_{1}, h_{2}, h_{2}, \ldots, h_{k}, 1,1, \ldots, 1,1, \ldots, 1, x_{1}, x_{2}, \ldots, x_{k}\right) \\
= & \beta\left(n, h_{1}, h_{2}, \ldots, h_{k} ; x_{1}, x_{2}, \ldots, x_{k}\right)
\end{aligned}
$$


(II) Extended Bernoulli numbers

$a_{1}=a_{2}=\ldots=a_{k}=m=i_{1}=i_{2}=\cdots=i_{k}=1$ and $x_{1}=x_{2}=\cdots=x_{k}=0$

Substituting, we get

$$
\begin{aligned}
& \left(n, h_{1}, h_{2}, h_{2}, \ldots, h_{k}, 1, \ldots, 1 ; 1, \ldots, 1,0,0, \ldots\right) \\
= & \beta\left(n, h_{1}, h_{2}, \ldots, h_{k}\right)
\end{aligned}
$$

(III) Extended Euler polynomials

when $a_{1}=a_{2}=\cdots=a_{k}=-1 ; i_{1}=i_{2}=\cdots=i_{k}=0$ and $m=2$

we are led to

$$
\begin{aligned}
& P\left(n, h_{1}, h_{2}, h_{2}, \ldots, h_{k},-1,-1, \ldots,-1,2,0, \ldots, 0, x_{1}, x_{2}, \ldots x_{k}\right) \\
= & \epsilon\left(n, h_{1}, h_{2}, \ldots, h_{k} ; x_{1}, x_{2}, \ldots x_{k}\right)
\end{aligned}
$$

(IV) Extended Euler numbers

Putting

$$
\begin{aligned}
& a_{1}=a_{2}=\cdots=a_{k}=-1, i_{1}=i_{2}=\ldots i_{k}=0 \\
& m=2 \text { and } x_{1}=x_{2}=\cdots=x_{k}=0
\end{aligned}
$$

we get

$$
\begin{aligned}
& P\left(n, h_{1}, h_{2}, \ldots, h_{k},-1,-1 \ldots-1,2,0, \ldots, 0,0, \ldots, 0\right) \\
= & \epsilon\left(n, h_{1}, h_{2}, \ldots, h_{k}\right)
\end{aligned}
$$

(V) Extended Eulerian polynomials

Yet another interesting special case of the polynomial set would occur when we let

$$
\begin{aligned}
& i_{1}=i_{2}=\ldots=i_{k}=0 \text { and } m=1 . \text { Thus we obtain } \\
& P\left(n, h_{1}, h_{2}, h_{2} \ldots, h_{k}, a_{1}, a_{2}, \ldots, a_{k}, 1,0, \ldots, 0, x_{1}, x_{2}, \ldots, x_{k}\right) \\
= & \frac{1}{\left(1-a_{1}\right) \times\left(1-a_{2}\right) \cdots\left(1-a_{k}\right)} H\left(n, h_{1}, h_{2}, \ldots, h_{k}, a_{1}, a_{2}, \ldots, a_{k}, x_{1}, x_{2}, \ldots, x_{k}\right)
\end{aligned}
$$

(VI) Extended Eulerian numbers

\section{Letting}

$$
\begin{aligned}
i_{1}= & i_{2}=\ldots=i_{k}=0 \text { and } m=1 \text { and } x_{1}=x_{2}=\ldots x_{k}=0, \quad \text { we have } \\
& P\left(n, h_{1}, h_{2}, \ldots, h_{k}, a_{1}, a_{2}, \ldots, a_{k}, 1,0, \ldots, 0,0,0,0, \ldots, 0\right) \\
= & \frac{1}{\left(1-a_{1}\right)\left(1-a_{2}\right) \cdots\left(1-a_{k}\right)} \times H\left(n, h_{1}, h_{2}, \ldots, h_{k}, a_{1}, a_{2}, \ldots, a_{k}\right)
\end{aligned}
$$

the above extended Bernoulli, Euler and Eulerian polynomials and their corresponding numbers are due to Carlitz [2].

In the present paper, we obtain numerous properties of the polynomials and numbers defined above. These properties are of an algebraic nature and for the most parts are generalizations of the corresponding properties of the Bernoulli, Euler and Eulerian polynomials and numbers. 


\section{Addition Theorems}

$$
\begin{aligned}
& \frac{2^{2}\left(i_{1}+i_{2}+\cdots+i_{k}\right)}{m^{2}} \\
& \times \sum_{c d=n} P\left(c, h_{1}, h_{2}, \ldots, h_{k}, a_{1}, a_{2}, \ldots, a_{k}, m, i_{1}, i_{2}, \ldots, i_{k}, 2 x_{1}, 2 x_{2}, \ldots, 2 x_{k}\right) \\
& \times \sum_{c d=n} P\left(d, h_{1}, h_{2}, \ldots, h_{k},-a_{1},-a_{2}, \ldots, a_{k}, m, i_{1}, i_{2}, \ldots, i_{k}, 2 y_{1}, 2 y_{2}, \ldots, 2 y_{k}\right) \\
= & P\left(n, 2 h_{1}, 2 h_{2}, \ldots, 2 h_{k}, a_{1}^{2}, a_{2}^{2}, \ldots, a_{k}^{2}, 2 i_{2}, \ldots, 2 i_{k}, x_{1}+y_{1}, x_{2}+y_{2}, \ldots, x_{k}+y_{k}\right)
\end{aligned}
$$

\section{Proof. Since}

$$
\begin{aligned}
& \frac{m^{2}\left(\frac{h_{1}}{m} \log \mathcal{G}(s)\right)^{i_{1}}\left(\frac{h_{2}}{m} \log \mathcal{G}(s)\right)^{i_{2}} \cdots\left(\frac{h_{k}}{m} \log \mathcal{G}(s)\right)^{i_{k}}}{\left((\mathcal{G}(s))^{h_{1}}-a_{1}\right)\left((\mathcal{G}(s))^{h_{2}}-a_{2}\right) \cdots\left((\mathcal{G}(s))^{h_{k}}-a_{k}\right)} \times\left((\mathcal{G}(s))^{2 h_{1} x_{1}+2 h_{2} x_{2}+\cdots+2 h_{k} x_{k}}\right. \\
& \times \frac{m^{2}\left(\frac{h_{1}}{m} \log \mathcal{G}(s)\right)^{i_{1}}\left(\frac{h_{2}}{m} \log \mathcal{G}(s)\right)^{i_{2}} \cdots\left(\frac{h_{k}}{m} \log \mathcal{G}(s)\right)^{i_{k}}}{\left((\mathcal{G}(s))^{h_{1}}+a_{1}\right)\left((\mathcal{G}(s))^{h_{2}}+a_{2}\right) \cdots\left((\mathcal{G}(s))^{h_{k}}+a_{k}\right)} \times\left((\mathcal{G}(s))^{2 h_{1} y_{1}+2 h_{2} y_{2}+\cdots+2 h_{k} y_{k}}\right. \\
= & m^{2} \frac{m^{2}\left(\frac{h_{1}}{m} \log \mathcal{G}(s)\right)^{2 i_{1}}\left(\frac{h_{2}}{m} \log \mathcal{G}(s)\right)^{2 i_{2}} \cdots\left(\frac{h_{k}}{m} \log \mathcal{G}(s)\right)^{2 i_{k}}}{\left((\mathcal{G}(s))^{2 h_{1}}-a_{1}^{2}\right)\left((\mathcal{G}(s))^{2 h_{2}}-a_{2}^{2}\right) \cdots\left((\mathcal{G}(s))^{2 h_{k}}-a_{k}^{2}\right.} \times(\mathcal{G}(s))^{2 h_{1}\left(x_{1}+y_{1}\right)+\cdots+2 h_{k}\left(x_{k}+y_{k}\right)}
\end{aligned}
$$

In the light of (3.1), after little simplification, we get

$$
\begin{aligned}
& \quad \sum_{n=1}^{\infty} P\left(c, h_{1}, h_{2}, \ldots, h_{k}, a_{1}, a_{2}, \ldots, a_{k}, m, i_{1}, i_{2}, \ldots, i_{k}, 2 x_{1}, 2 x_{2}, \ldots 2 x_{k}\right) \\
& \quad \times \sum_{n=1}^{\infty} P\left(d, h_{1}, h_{2}, \ldots h_{k},-a_{1},-a_{2}, \ldots,-a_{k}, m, i_{1}, i_{2}, \ldots, i_{k}, 2 y_{1}, 2 y_{2}, \ldots, 2 y_{k}\right) \\
& \quad=\frac{m^{2}}{2^{2 i_{1}+2 i_{2}+\cdots+2 i_{k}} \times} \\
& \sum_{n=1}^{\infty} P\left(c, 2 h_{1}, 2 h_{2}, \ldots, 2 h_{k}, a_{1}^{2}, a_{2}^{2}, \ldots, a_{k}^{2}, 2 i_{1}, 2 i_{2}, \ldots, 2 i_{k}, x_{1}+y_{1}, x_{2}+y_{2}, \ldots, x_{k}+y_{k}\right) \\
& \text { whcih } \operatorname{completes} \text { the proof. } \\
& \quad \frac{2^{2\left(i_{1}+i_{2}+\cdots i_{k}\right)}}{m^{2}} \sum_{c d=n} P\left(c, h_{1}, h_{2}, \ldots, h_{k}, a_{1}, a_{2}, \ldots, a_{k}, m, i_{1}, i_{2}, \ldots, i_{k}, 2 x_{1}, 2 x_{2}, \ldots, 2 x_{k}\right) \\
& \quad \times \sum_{c d=n} P\left(d, h_{1}, h_{2}, \ldots, h_{k}, a_{1}, a_{2}, \ldots, m, i_{1}, i_{2}, \ldots, i_{k}, y_{1}, y_{2}, \ldots, y_{k-1}, 0\right) \\
& =P\left(n, 2 h_{1}, 2 h_{2}, \ldots, 2 h_{k}, a_{1}^{2}, a_{2}^{2}, \ldots, a_{k}^{2}, 2 i_{1}, 2 i_{2}, \ldots, 2 i_{k}, x_{1}+y_{1}, x_{2}+y_{2}, \ldots, x_{k}+0\right)
\end{aligned}
$$

Proof is similar to the proof of (4.1) 


\section{Multiplication Theorems}

$$
\begin{aligned}
& \sum_{r_{1}=0}^{\mu_{1}-1} \sum_{r_{2}=0}^{\mu_{2}-1} \ldots \sum_{r_{k}=0}^{\mu_{k}-1} \frac{1}{a_{1}^{r_{1}} a_{2}^{r_{2}} \ldots a_{k}^{r_{k}}} \\
& \times P\left(n, h_{1}, \mu_{1}, \ldots, h_{k}, \mu_{k}, a_{1}^{\mu_{1}}, \ldots, a_{k}^{\mu_{k}}, m, i_{1}, i_{2}, \ldots, i_{k}, x_{1}+\frac{r_{1}}{\mu_{1}}, \ldots, x_{k}+\frac{r_{k}}{\mu_{k}}\right) \\
= & \frac{\mu_{1}^{i_{2}} \cdot \mu_{2}^{i_{2}} \cdots \mu_{k}^{i_{k}}}{a_{1}^{\mu_{1}-1} \cdot a_{2}^{\mu_{2}-1} \cdots a_{k}^{\mu_{k}-1}} \\
& \times P\left(n, h_{1}, h_{2}, \ldots, h_{k}, a_{1}, a_{2}, \ldots, a_{k}, m, i_{1}, i_{2}, \ldots, i_{k}, \mu_{1} x_{1}, \mu_{2} x_{2}, \ldots, \mu_{k} x_{k}\right)
\end{aligned}
$$

where and throughout this investigation, $\mu_{1}, \mu_{2}, \ldots, \mu_{k}$ are positive integers.

Proof. By virtue of generating relation (3.1), we have

$$
\begin{aligned}
& \sum_{n=1}^{\infty} \frac{P\left(n, h_{1}, h_{2}, \ldots, h_{k}, a_{1}, a_{2}, \ldots, a_{k}, m, i_{1}, i_{2}, \ldots, i_{k}, \mu_{1} x_{1}, \mu_{2} x_{2}, \ldots, \mu_{k} x_{k}\right)}{n^{s}} \\
= & \frac{m^{2}\left(\frac{h_{1}}{m} \log \mathcal{G}(s)\right)^{i_{1}}\left(\frac{h_{2}}{m} \log \mathcal{G}(s)\right)^{i_{2}} \ldots\left(\frac{h_{k}}{m} \log \mathcal{G}(s)\right)^{i_{k}}}{\left((\mathcal{G}(s))^{h_{1}}-a_{1}\right)\left((\mathcal{G}(s))^{h_{2}}-a_{2}\right) \cdots\left((\mathcal{G}(s))^{h_{k}}-a_{k}\right)}(\mathcal{G}(s))^{h_{1} \mu_{1} x_{1}+h_{2} \mu_{2} \mu_{2} x_{2}+\cdots+h_{k} \mu_{k} x_{k}} \\
= & a_{1}^{\mu_{1}-1} a_{2}^{\mu_{2}-1} \ldots a_{k}^{\mu_{k}-1} \times \frac{m^{2}\left(\frac{h_{1}}{m} \log \mathcal{G}(s)\right)^{i_{1}}\left(\frac{h_{2}}{m} \log \mathcal{G}(s)\right)^{i_{2}} \ldots\left(\frac{h_{k}}{m} \log \mathcal{G}(s)\right)^{i_{k}}}{\left((\mathcal{G}(s))^{h_{1} \mu_{1}}-a_{1}^{\mu_{1}}\right) \cdots\left((\mathcal{G}(s))^{h_{k} \mu_{k}}-a_{k}^{\mu_{k}}\right)} \\
& \times \sum_{r_{1}=0}^{\mu_{1}-1} \frac{(\mathcal{G}(s))^{r_{1} h_{1}}}{a_{1}^{r_{1}}} \cdots \sum_{r_{k}=0}^{\mu_{k}-1} \frac{(\mathcal{G}(s))^{r_{k} h_{k}}}{a_{k}^{r_{k}}} \\
= & a_{1}^{\mu_{1}-1} a_{2}^{\mu_{2}-1} \cdots a_{k}^{\mu_{k}-1} \times \frac{m^{2}\left(\frac{h_{1}}{m} \log \mathcal{G}(s)\right)^{i_{1}}\left(\frac{h_{2}}{m} \log \mathcal{G}(s)\right)^{i_{2}} \ldots\left(\frac{h_{k}}{m} \log \mathcal{G}(s)\right)^{i_{k}}}{\left((\mathcal{G}(s))^{h_{1} \mu_{1}}-a_{1}^{\mu_{1}}\right) \cdots\left((\mathcal{G}(s))^{h_{k} \mu_{k}}-a_{k}^{\mu_{k}}\right)} \\
& \times \sum_{r_{1}=0}^{\mu_{1}-1}(\mathcal{G}(s))^{\left(x_{1}+\frac{r_{1}}{\mu_{1}}\right) \mu_{1} h_{1}} \ldots \sum_{r_{k}=0}^{\mu_{k}-1}(\mathcal{G}(s))^{\left(x_{k}+\frac{r_{1}}{\mu_{k}}\right) \mu_{k} h_{k}}
\end{aligned}
$$

By an appeal to (3.1), the result would follow immediatedly.

\section{Theorem 5.2.}

$$
\begin{aligned}
& a_{1}^{\mu_{1}} a_{2}^{\mu_{2}} \cdots a_{k}^{\mu_{k}} \cdot p_{1}^{i_{1}} p_{2}^{i_{2}} \cdots p_{k}^{i_{k}} \sum_{r_{1}=0}^{\mu_{1}-1} \cdots \sum_{r_{k}=0}^{\mu_{k}-1} \frac{1}{a_{1}^{r_{1} p_{1}} a_{2}^{r_{2} p_{2}} \cdots a_{k}^{r_{k} p_{k}}} \\
& \times P\left(n, h_{1} \mu_{1}, \ldots h_{k} \mu_{k}, a_{1}^{\mu_{1}}, \ldots a_{k}^{\mu_{k}}, m, i_{1}, \ldots i_{k}, \frac{x_{1}}{\mu_{1}}+\frac{r_{1} p_{1}}{\mu_{1}}, \ldots, \frac{x_{k}}{\mu_{k}}+\frac{r_{k} p_{k}}{\mu_{k}}\right. \\
= & \mu_{1}^{i_{1}} \mu_{2}^{i_{2}} \ldots \mu_{k}^{i_{k}} a_{1}^{p_{1}} a_{2}^{p_{2}} \ldots a_{k}^{p_{k}} \sum_{e_{1}=0}^{p_{1}-1} \ldots \sum_{e_{k}=0}^{p_{k}-1} \frac{1}{a_{1}^{\mu_{1} e_{1}} a_{k}^{\mu_{k} e_{k}}} \\
& \times P\left(n, p_{1} h_{1}, \ldots, p_{k} h_{k}, a_{1}^{p_{1}}, \ldots, a_{k}^{p_{k}}, m, i_{1}, \ldots, i_{k}, \frac{x_{1}}{p_{1}}+\frac{e_{1} \mu_{1}}{p_{1}}, \cdots, \frac{x_{k}}{p_{k}}+\frac{e_{k} \mu_{k}}{p_{k}}\right)
\end{aligned}
$$


proof is similar to the proof of (5.1)

\section{Finite Difference Formula}

Norlund's operator is defined by the relation

$$
\triangle_{W} U(x)=\frac{U(x+w)-U(x)}{w}
$$

This symbol has the advantage that

$$
\operatorname{Lim}_{w \rightarrow 0} \Delta U(x)=D U(x), D=\frac{d}{d x}
$$

The definition of the operator $\underset{W}{\nabla}$ is given

$$
\underset{W}{\nabla} U(x)=\frac{1}{2}[U(x)+U(x+w)]
$$

If $w=1$, it is convenient to write $\Delta$ instead of $\Delta$ and $\nabla$ instead $\nabla$, Again, consider $f(x, y)$, where $x$ and $y$ are regarded as independent variables. We then define partial difference quotients with respect to $X$ and $Y$ by

$$
\underset{W^{x}}{\Delta} f(x, y)=[f(x+w, y)-f(x, y)] / W
$$

and

$$
\underset{h^{y}}{\Delta} f(x, y)=[f(x, y+h)-f(x, y)] / h
$$

These symbols have the advantage that

$$
\operatorname{Lim}_{W \rightarrow 0} \underset{W^{x}}{\Delta} f(x, y)=D_{x} f(x, y)
$$

and

$$
\operatorname{Lim}_{h \rightarrow 0} \underset{h^{y}}{ } f(x, y)=D_{y} f(x, y)
$$

Likewise

$$
\underset{W^{x}}{\nabla} f(x, y)=\frac{1}{2}[f(x, y)+f(x+w, y)]
$$

and

$$
\underset{h^{y}}{\nabla} f(x, y)=\frac{1}{2}[f(x, y)+f(x, y+h)]
$$

Similarly, the above operators can be defined for multivariate function $f\left(x_{1}, x_{2}, \ldots, x_{k}\right)$ as under

$$
\underset{W_{1}^{x_{1}}}{\Delta} f\left(x_{1}, x_{2}, \ldots, x_{k}\right)=\left[\left[f\left(x_{1}+w_{1}, x_{2}, \ldots, x_{k}\right)-f\left(x_{1}, x_{2}, \ldots, x_{k}\right)\right] / W_{1}\right.
$$




$$
\underset{W_{2}^{x_{2}}}{\Delta} f\left(x_{1}, x_{2}, \ldots, x_{k}\right)=\left[f\left(x_{1}, x_{2}+w_{2}, x_{3}, \ldots, x_{k}\right)-f\left(x_{1}, x_{2}, \ldots, x_{k}\right)\right] 1 / W_{2}
$$

and similarly

$$
\underset{W_{k}^{x_{k}}}{\Delta} f\left(x_{1}, x_{2}, \ldots, x_{k}\right)=\left[\left[f\left(x_{1}, x_{2}, \ldots, x_{k}+w_{k}\right)-f\left(x_{1}, x_{2}, \ldots, x_{k}\right)\right] / W_{k}\right.
$$

These symbols have advantage that

$$
\begin{aligned}
& \operatorname{Lim}_{W_{1} \rightarrow 0} \underset{W_{2}^{x_{2}}}{\Delta} f\left(x_{1}, x_{2}, \ldots, x_{k}\right)=D_{x_{1}} f\left(x_{1}, x_{2}, \ldots, x_{k}\right) \\
& \operatorname{Lim}_{W_{2} \rightarrow 0} \underset{W_{2}^{x_{2}}}{\Delta} f\left(x_{1}, x_{2}, \ldots, x_{k}\right)=D_{x_{2}} f\left(x_{1}, x_{2}, \ldots, x_{k}\right)
\end{aligned}
$$

and

$$
\operatorname{Lim}_{W_{k} \rightarrow 0} \underset{W_{k}^{x_{k}}}{\Delta} f\left(x_{1}, x_{2}, \ldots, x_{k}\right)=D_{x_{k}} f\left(x_{1}, x_{2}, \ldots, x_{k}\right)
$$

As above, we can define the $\nabla$ for $f\left(x_{1}, x_{2}, \ldots, x_{k}\right)$

$$
\begin{aligned}
\underset{W_{1}^{x_{1}}}{\nabla} f\left(x_{1}, x_{2}, \ldots, x_{k}\right) & =\frac{1}{2}\left[f\left(x_{1}, x_{2}, \ldots, x_{k}\right)+f\left(x_{1}+w_{1}, x_{2}, x_{3}, \ldots, x_{k}\right)\right] \\
\underset{W_{2}^{x_{2}}}{\nabla} f\left(x_{1}, x_{2}, \ldots, x_{k}\right) & =\frac{1}{2}\left[f\left(x_{1}, x_{2} \ldots, x_{l}\right)+f\left(x_{1}, x_{2}+w_{2}, x_{3}, \ldots, x_{k}\right)\right]
\end{aligned}
$$

Similarly,

$$
\underset{W_{k}^{x_{k}}}{\Delta} f\left(x_{1}, x_{2}, \ldots, x_{k}\right)=\frac{1}{2}\left[f\left(x_{1}, x_{2}, \ldots, x_{k}\right)+f\left(x_{1}, x_{2}, \ldots, x_{k}+w_{k}\right)\right] .
$$

operating on (3.1) with $\Delta_{x_{1}}$ we find that

$$
\begin{aligned}
& \sum_{n=1}^{\infty} \Delta_{x} \frac{P\left(n, h_{1}, h_{2}, \ldots, h_{k}, a_{1}, \ldots, a_{k}, m, i_{1}, i_{2}, \ldots, i_{l}, x_{1}, x_{2}, \ldots, x_{k}\right)}{n^{s}} \\
& \frac{m^{2}\left(\frac{h_{1}}{m} \log \mathcal{G}(s)\right)^{i_{1}}\left(\frac{h_{2}}{m} \log \mathcal{G}(s)\right)^{i_{2}} \ldots\left(\frac{h_{k}}{m} \log \mathcal{G}(s)\right)^{i_{k}}}{\left((\mathcal{G}(s))^{h_{1}}-a_{1}\right)\left((\mathcal{G}(s))^{h_{2}}-a_{2} \ldots\left((\mathcal{G}(s))^{h_{k}}-a_{k}\right)\right.} \times\left(( \mathcal { G } ( s ) ) ^ { h _ { 1 } x _ { 1 } + h _ { 2 } x _ { 2 } + \cdots + h _ { k } x _ { k } } \left(\mathcal{G}(s)^{h_{1}}\right.\right. \\
= & \frac{m^{2}\left(\frac{h_{1}}{m} \log \mathcal{G}(s)\right)^{i_{1}}\left(\frac{h_{2}}{m} \log \mathcal{G}(s)\right)^{i_{2}} \ldots\left(\frac{h_{k}}{m} \log \mathcal{G}(s)\right)^{i_{k}}}{\left((\mathcal{G}(s))^{h_{1}}-a_{1}\right)\left((\mathcal{G}(s))^{h_{2}}-a_{2}\right) \cdots\left((\mathcal{G}(s))^{h_{k}}-a_{k}\right)} \times(\mathcal{G}(s))^{h_{1} x_{1}+h_{2} x_{2}+\cdots+h_{k} x_{k}} \\
= & \frac{\left((\mathcal{G}(s))^{h_{1}}-1\right) m^{2}\left(\frac{h_{1}}{m} \log \mathcal{G}(s)\right)^{i_{1}} \ldots\left(\frac{h_{k}}{m} \log \mathcal{G}(s)\right)^{i_{k}}}{\left((\mathcal{G}(s))^{h_{1}}-a_{1}\right)\left(\left(\mathcal{G}(s)^{h_{2}}-a_{2}\right) \ldots\left((\mathcal{G}(s))^{h_{k}}-a_{k}\right)\right.} \times(\mathcal{G}(s))^{h_{1} x_{1}+h_{2} x_{2}+\cdots+h_{k} x_{k}}
\end{aligned}
$$

In view of (3.1) and the definition of $\tau_{x}(n)$ in [2], the above expression immediately yields

$$
\begin{aligned}
& \Delta_{x_{1}} P\left(n, h_{1}, h_{2}, \ldots, h_{k}, a_{1}, \ldots, a_{2}, \ldots, a_{k}, m, i_{1}, i_{2}, \ldots, i_{k}, x_{1}, x_{2}, \ldots, x_{k}\right) \\
= & \sum_{c d=n} \tau_{h_{1}}(c) P\left(d, h_{1}, h_{2}, \ldots, h_{k}, a_{1}, a_{2}, \ldots, a_{k}, m, i_{1}, i_{2}, \ldots, i_{k}, x_{1}, x_{2}, \ldots, x_{k}\right) \\
& P\left(n, h_{1}, h_{2}, \ldots, h_{k}, a_{1}, a_{2}, \ldots, a_{k}, m, i_{1}, i_{2}, \ldots, i_{k}, x_{1}, x_{2}, \ldots, x_{k}\right)
\end{aligned}
$$


Similarly, operting on (3.1) with $\Delta_{x_{k}}$ we intuitively obtain

$$
\begin{aligned}
& \Delta_{x_{k}} P\left(n, h_{1}, h_{2}, \ldots, h_{k}, a_{1}, a_{2}, \ldots, a_{k}, m, i_{1}, i_{2}, \ldots, i_{k}, x_{1}, x_{2}, \ldots, x_{k}\right) \\
= & \sum_{c d=n} \tau_{h_{k}}(c) P\left(d, h_{1}, h_{2}, \ldots, h_{k}, a_{1}, a_{2}, \ldots, a_{k}, m, i_{1}, i_{2}, \ldots, i_{k}, x_{1}, x_{2}, \ldots, x_{k}\right) \\
& -P\left(n, h_{1}, h_{2}, \ldots, h_{k}, a_{1}, a_{2}, \ldots, a_{k}, m, i_{1}, i_{2}, \ldots, i_{k}, x_{1}, x_{2}, \ldots x_{k}\right)
\end{aligned}
$$

Furthermore, operating $\nabla_{x_{1}}$ on the generating relation (3.1) we have

$$
\begin{aligned}
& \sum_{n=1}^{\infty} \nabla_{x_{1}} \frac{P\left(n, h_{1}, h_{2}, \ldots, h_{k}, a_{1}, a_{2}, \ldots a_{k}, m, i_{1}, i_{2}, \ldots, i_{k}, x_{1}, x_{2}, \ldots x_{k}\right)}{n^{s}} \\
& \frac{\frac{1}{2}\left(\mathcal{G}(s)^{h_{k}}+1\right) m^{2}\left(\frac{h_{1}}{m} \log \mathcal{G}(s)\right)^{i_{1}} \cdots\left(\frac{h_{k}}{m} \log \mathcal{G}(s)\right)^{i_{k}}}{\left.\left((\mathcal{G}(s))^{h_{1}}-a_{1}\right)\left((\mathcal{G}(s))^{h_{2}}-a_{2}\right) \cdots((\mathcal{G})(s))^{h_{k}}-a_{k}\right)} \times\left((\mathcal{G}(s))^{h_{1} x_{1}+h_{2} x_{2}+\cdots+h_{k} x_{k}}\right. \\
= & \sum_{n=1}^{\infty} \frac{1}{2}\left(\frac{\tau_{h_{1}}(n)}{n^{s}}+1\right) \\
& \times \sum_{n=1}^{\infty} \frac{P\left(n, h_{1}, h_{2}, \ldots, h_{k}, a_{1}, \ldots, a_{k}, m, i_{1}, i_{2}, \ldots, i_{k}, x_{1}, x_{2}, \ldots, x_{k}\right)}{n^{s}}
\end{aligned}
$$

In the light of generatin relation (3.1) and Carlitz [2]. Finally, we arrive at

$$
\begin{aligned}
& \nabla_{x_{1}} P\left(n, h_{1}, h_{2}, \ldots, h_{k}, a_{1}, \ldots, a_{k}, m, i_{1}, i_{2}, \ldots, i_{k}, x_{1}, x_{2}, \ldots, x_{k}\right) \\
= & \frac{1}{2} \sum_{c d=n} \tau_{h_{1}}(c) P\left(d, h_{1}, h_{2}, \ldots, h_{k}, a_{1}, a_{2}, \ldots, a_{k}, m, i_{1}, i_{2}, \ldots, i_{k}, x_{1}, x_{2}, \ldots, x_{k}\right) \\
& +\frac{1}{2} P\left(n, h_{1}, h_{2}, \ldots, h_{k}, a_{1}, \ldots, a_{k}, m, i_{1}, i_{2}, \ldots, i_{k}, x_{1}, x_{2}, \ldots, x_{k}\right)
\end{aligned}
$$

Now finally we obtain the expression for $\nabla_{x_{k}} P$ which is similar to the expression $\nabla_{x_{1}} P$ by operating $\nabla_{x_{k}}$ on the generating relation (3.1) as

$$
\begin{aligned}
& \nabla_{x_{k}} P\left(n, h_{1}, h_{2}, \ldots, h_{k}, a_{1}, \ldots, a_{k}, m, i_{1}, i_{2}, \ldots, i_{k}, x_{1}, x_{2}, \ldots, x_{k}\right) \\
= & \frac{1}{2} \sum_{c d=n} \tau_{h_{1}}(c) P\left(d, h_{1}, h_{2}, \ldots, h_{k}, a_{1}, a_{2}, \ldots, a_{k}, m, i_{1}, i_{2}, \ldots, i_{k}, x_{1}, x_{2}, \ldots, x_{k}\right) \\
& +\frac{1}{2} P\left(n, h_{1}, h_{2}, \ldots, h_{k}, a_{1}, \ldots, a_{k}, m, i_{1}, i_{2}, \ldots, i_{k}, x_{1}, x_{2}, \ldots, x_{k}\right)
\end{aligned}
$$

\section{References}

[1] L. Carlitz, "Eulerian numbers and polynomials," Math Magazine, 33(1959), 247-260.

[2] L. Carlitz, "Extended Bernoulli and Eulerian numbers," Duke Math, J., 31(1964), 667-689.

[3] L. Carlitz, "Enumeration of sequences by rises and falls; a refinement of the Simon New comb's Problem," Duke Math. J., 39(1972), 267-280.

[4] L. Carlitz and R. Scovilje, "Generalized Eulerian numbers: Combinatorial applications", J.fur die reine und angewandte mathematik, 265(1874), 110-137. 
[5] J. F. Dillon and D. F. Roselle, "Simon Newcomb's problems," SIAM J. Appl. Math., 17(1969), 1086-1093.

[6] D. Foata and M. P. Schutzenberger, Theorie geometrique des polynomes Eulerians, Leture Notes in Math. 138. Berlin, Heidelberg, New York: Springer-Verlag, 1970.

[7] S. N. Singh and B. K. Rai, "Properties of some extended Bernoulli and Euler polynomials", The Fibanacci Qua., 21(3)(1983), 162-172.

[8] S. N. Singh and V. S. Rai, "Certain Properties of extended Euler and Bernoulli polynomials," Tamkang Journal of Mathematics, 16(4)(1985), 1-12.

[9] H. M. Srivastava and H. L. Manocha, A treatise on generating functions, Wiley/Halsted, New York, 1984.

Department of Mathematics and Statistics, Avadh University, Faizabad-224001, (U.P), INDIA. 